die Kompression des Nervus medianus im Bereich des Handgelenkes hervorgerufen. Es ist eines der häufigsten Nervenkompressionssyndrome. Aufgrund der Symptomatik kann folgende Stadieneinteilung für diese Erkrankung getroffen werden:

1. Uncharakteristische Beschwerden im Handbereich.

2. Lokalisierung zunehmender Beschwerden im Medianusinnervationsgebiet.

3. Starke Funktionsbeeinträchtigung der Finger verbunden mit erheblichen Schmerzen; Schwellung auf der Handgelenksbeugeseite.

4. Daumenballenatrophie, trophische Störung, Sensibilitätsverlust.

Neben zahlreichen anderen Ursachen kann eine unspezifische oder spezifische Tenosynovitis ein Carpaltunnelsyndrom auslösen. Die teilweise Resektion des Ligamentum carpi transversum verbunden mit einer Tenosynovektomie bringt sofort Schmerzfreiheit.

Ähnliche Symptome werden durch die Ulnariskompression im Bereich des Sulcus nervi ulnaris hervorgerufen. Ursache ist vorwiegend die degenerative oder entzündliche Veränderung des Ellenbogengelenks. Auch hier führt die konservative Behandlung nicht weiter, die operative Verlagerung des Nerven in die Ellenbeuge kann jedoch eine erhebliche Besserung der Beschwerden erzielen.

Für die Wissenschaftliche Ausstellung von A.Gregl (a.G.): 9. Beitrag zur Mammographie und Lymphographie wurde kein Manuskript eingereicht.

\title{
10. Demonstration eines neuen Modells des Cholangiometers
}

\section{H. BRÜCKE}

Landeskrankenhaus Leoben/Osterreich

Das Cholangiometer nach Brücke stellt ein einfaches, klinisch brauchbares Gerät zur Messung von Druck und Abflußgeschwindigkeit im Gallengang und zur Kalibrierung der Papille dar. Die Messung geschieht mit Hilfe eines Meßhahns bei konstant gehaltenen Druck im Zuflußteil und im Abflußteil (Standarddruck).

Die Neukonstruktion des Hahns garantiert durch eine Innenschicht aus Teflon gute Abdichtung und leichte Einstellung des Meßhahns. 